\title{
Anomalous Forbush effects from sources far from Sun center
}

\author{
E. Eroshenko ${ }^{1}$, A. Belov ${ }^{1}$, H. Mavromichalaki ${ }^{2}$, V. Oleneva ${ }^{1}$, \\ A. Papaioannou ${ }^{2}$ and V. Yanke ${ }^{1}$ \\ ${ }^{1}$ Pushkov Institute of Terrestrial Magnetism, Ionosphere and Radio Wave Propagation \\ Russian Academy of Sciences (IZMIRAN), 142190 Troitsk, \\ Moscow region, Russia \\ email: abelov@izmiran.ru \\ ${ }^{2}$ Nuclear and Particle Physics Section, Physics Department, University of Athens, \\ Panepistimiopolis, Zografos GR-15783, Athens, Greece
}

\begin{abstract}
The Forbush effects associated with far western and eastern powerful sources on the Sun that occurred on the background of unsettled and moderate interplanetary and geomagnetic disturbances have been studied by data from neutron monitor networks and relevant measurements of the solar wind parameters. These Forbush effects may be referred to a special sub-class of events, with the characteristics like the event in July 2005, and incorporated by the common conditions: absence of a significant disturbance in the Earth vicinity; absence of a strong geomagnetic storm; slow decrease of cosmic ray intensity during the main phase of the Forbush effect. General features and separate properties in behavior of density and anisotropy of $10 \mathrm{GV}$ cosmic rays for this subclass are investigated.
\end{abstract}

Keywords. elementary particles, Sun: activity, interplanetary medium, solar-terrestrial relations, coronal mass ejections (CMEs), shock waves.

\section{Introduction}

We consider the Forbush effect (FE) as a result of influence of interplanetary disturbance on the galactic cosmic rays $(\mathrm{CR})$ : it is the response of cosmic rays to the propagating disturbance including precursors (pre-increase and pre-decrease in CR variations), $\mathrm{CR}$ intensity decrease as the main phase (FD), and the recovery phase while the Earth exits a disturbance area (Belov et al. 2003a, Belov et al. 2007). Usually FE is observed simultaneously with the interplanetary magnetic field (IMF) increase. Cosmic ray intensity decrease (FD) during the FE is created in a separate region of interplanetary space where the particle access from outside is difficult. The stronger the IMF created in this special area, the wider is rigidity diapason of CR exposed to this effect, the stronger the CR modulation. As a rule, the deep Forbush decreases correspond to the big increases of the IMF intensity. In Belov et al. (2005) we studied the exceptions to this rule where FEs with a magnitude more than $6 \%$ were created on the background of weak disturbances of the IMF. It was found that in a large fraction of the efficient events, the Earth is struck only by a peripheral area of the interplanetary disturbance and the main part misses the Earth, as a rule to the east. However, the event in July 2005 caused by the far western source on the Sun indicated analogous features of higher efficiency of a disturbance modulating influence on the CR (Papaioannou et al. 2008). In the present work we continue our study of such anomalous effects. Among all FEs we distinguished a subclass of events characterized by relatively unsettled interplanetary and geomagnetic conditions ( $\mathrm{IMF}<15 \mathrm{nT}, \mathrm{Kp}<6$ ), gradual decrease of the $\mathrm{CR}$ intensity on the main phase of $\mathrm{FE}$ and slow recovery phase. It turned out that all these events were caused by 


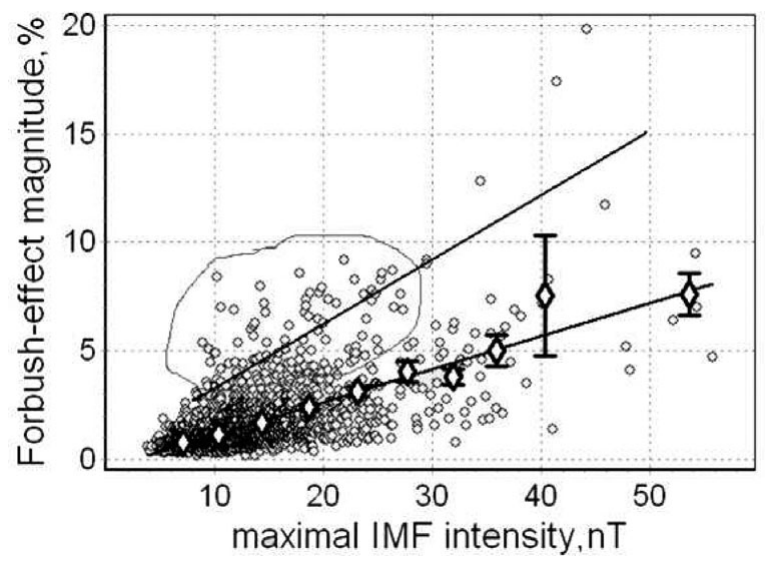

Figure 1. FE magnitude $\left(\mathrm{A}_{m}\right)$ vs the maximal IMF intensity $\mathrm{B}_{m}$. Diamonds are the $\mathrm{A}_{F}$ values averaged by the equal $\mathrm{B}_{m}$ intervals.

far eastern or western solar sources and have very characteristic features in a behavior of $\mathrm{CR}$ variations. The goal of our work is to analyze the subclasses of such anomalous FEs associated with far eastern and western solar flares, to study the variations of CR density and anisotropy during these effects and to compare and contrast the CR behavior for these two groups with the aim to use these results for diagnostics of the interplanetary space and near-Earth vicinity.

\section{Data and methods}

The database on the Forbush effects and interplanetary disturbances, created in IZMIRAN, has been used for the above study. This database includes as parameters of CR density (A0) and anisotropy (equatorial component of the first harmonic, Axy) and data on the solar wind, interplanetary magnetic field, solar data and geomagnetic activity indices Kp and Dst (Archive SPIDR Data Base, available from http://spidr.ngdc.noaa.gov). The updated measurements on GOES, and OMNI data base have been utilized from the web sites: http://www.ngdc.noaa.gov, http://omniweb.gsfc.nasa.gov, http://sec.ts.astro.it and CME data from http://lasco-www.nrl.navy.mil. The list of sudden storm commencements (SSC) has been also used (ftp://ftp.ngdc.noaa.gov) as a proxy for interplanetary shocks; the time of SSC generally defines the onset of FE. Density and anisotropy for $10 \mathrm{GV}$ CR was derived by the global survey method (Belov et al. 2005, Belov et al. 2007) over the period 1965-2006 by the hourly data from neutron monitor network (http://cr0.izmiran.rssi.ru/common/links.htm) and included into the IZMIRAN database (Asipenka et al. 2008). Normally, the stronger the interplanetary disturbance, the bigger is the $\mathrm{FE}$ amplitude $\left(\mathrm{A}_{F}\right)$ which has in total a linear dependence on $\mathrm{B}_{m}$ (Belov et al. 2001). Only the events separated by at least 24 hours have been taken for the analysis and only those where the IMF data provided has at least $80 \%$ coverage.

\section{Discussion of the results}

The well known relation between IMF intensity and FD magnitude (Belov et al., 2001) is obtained in the present work on the extremely large statistical base ( 2000 events).

In Fig. 1 one can see a dependence of $\mathrm{FE}$ magnitude $\left(\mathrm{A}_{F}\right)$ on the maximum IMF intensity $\mathrm{B}_{m}$. The majority of points are concentrated near the regression line, but there is 

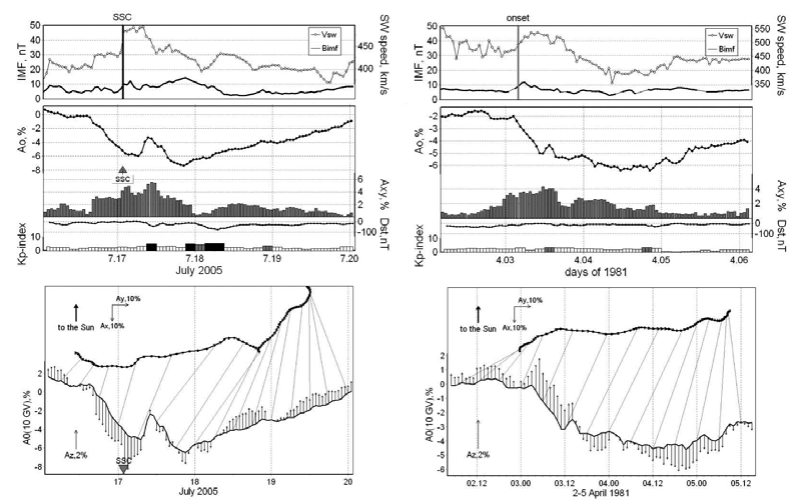

Figure 2. Examples of the FEs in July 2005 and April 1981 caused by the far western solar sources: IMF, solar wind, geomagnetic data (Kp-index and Dst variations), cosmic ray density and anisotropy (A0 and Axy) during the FEs associated with the western solar flares W79 and W52. In the bottom panels vector diagram of CR anisotropy (equatorial component Axy) and density (A0) are presented. Vertical vectors mean north-south component of the CR anisotropy. Thin lines connect equal time points in each 6 hours in vector diagram and density curves.

a group placed significantly higher than this line. A correlation coefficient of 0.67 obtained by the 1987 events shows a relation between $\mathrm{FE}$ magnitudes and $\mathrm{B}_{m}$, so the statistical character of this relation and the possibility to be essentially violated in some cases: large FEs observed under relatively low values of the IMF intensity, and on the contrary, relatively small FEs corresponded sometimes to large IMF intensity. We were interested in the events (circled group) where small and moderate values of IMF corresponded to large $\mathrm{A}_{F}$. It looks as a result of more effective influence of a disturbance on the CR. A degree of such the efficiency might be estimated as $\mathrm{K}_{F}=\mathrm{A}_{F} / \mathrm{B}_{m}, \% / \mathrm{nT}$.

This coefficient may be considered as a characteristic of the modulating ability of the associated IMF disturbance and it was one of the main parameters by which a selection of events for analysis was carried out. Our experience shows the events with $\mathrm{K}_{F}>0.36$ belong to a group of anomalous events when near-Earth measured IMF parameters could not provide the observable Forbush effect. Selection of the FEs by this parameter among events identified with associated solar sources gives the following distribution by the source longitudes within $60^{\circ}$ ranges.

\begin{tabular}{lccc}
\hline type & East (E90-E30) & Centre (E30-W30) & West (W30-W90) \\
\hline $\mathrm{K}_{F}>0.36$ anomalous & 14 & 10 & 9 \\
$\mathrm{~K}_{F}<0.36$ normal & 24 & 62 & 13 \\
\hline
\end{tabular}

One can see that in normal events the central sources dominate whereas anomalous FEs are mainly caused by extreme eastern or western sources. All anomalous FEs turned out to be of $>3 \%$ magnitude and occurred in relatively weakly disturbed interplanetary and geomagnetic conditions ( $\mathrm{IMF}<15 \mathrm{nT}, \mathrm{Kp}<6$ ), with gradual fall of $\mathrm{CR}$ intensity in the main phase of Forbush-effect. We compared the characteristics of $\mathrm{CR}$ variations during the FEs from western and eastern sources.

In Fig. 2 the examples of FEs in July 2005 (western source at the longitude W79 ${ }^{\circ}$ ) and in April 1981 (longitude W52 ${ }^{\circ}$ ) are presented. In both cases on the background of relatively quiescent interplanetary and geomagnetic conditions we observe FEs with the 

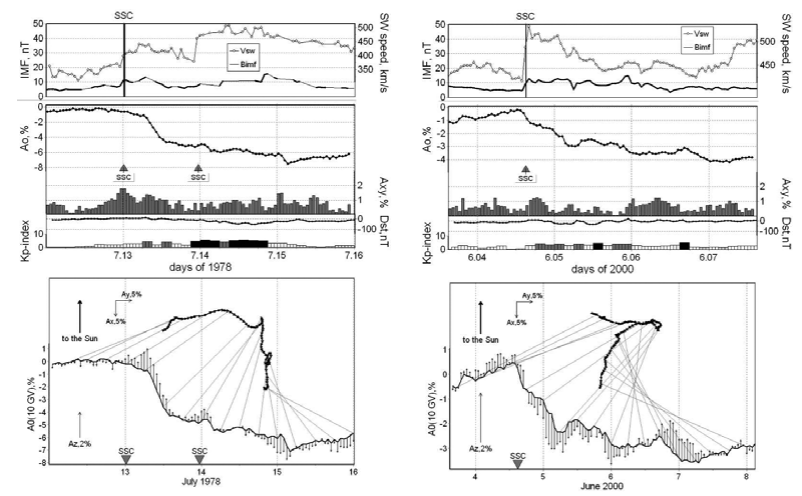

Figure 3. Parameters of the cosmic ray, interplanetary and geomagnetic activity during the FEs associated with far eastern solar flares: in July 1978 (E58) and June 2000 (E60). There designations are the same as in Fig 2.
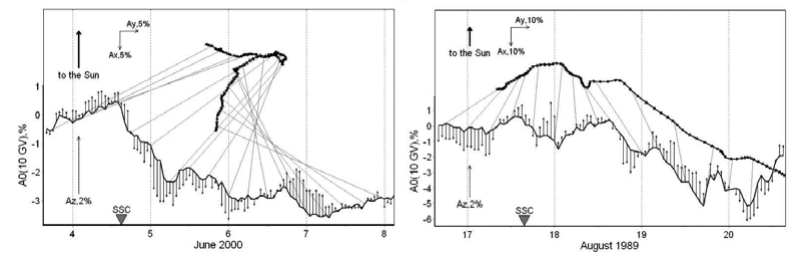

Figure 4. Vector diagrams for equatorial component of CR anisotropy Axy during the FEs associated with eastern (June, 2000, E60) and western (August 1989, W60) solar sources. The designations are the same as in Fig 2.

magnitude of $\mathrm{FD}>4 \%$ and significant increases of Axy, up to $4.6 \%$ in the main phase (whereas in normal events it doesn't exceed 2\%). The Axy increase started at once with the $\mathrm{FE}$ onset thereafter the direction of Axy vector varied very little and remained usually westward during the recovery phase.

In Fig. 3 we give the same presentation of different parameters for the FEs associated with far eastern solar sources. The difference in behavior of the vector Axy in these cases and in western events during the FEs is evident.

The magnitude of FEs caused by the eastern sources turned out to be a little less than for the western ones, but the vector of the equatorial component of CR anisotropy Axy shows quite different behavior by direction (see Fig. 4). For 9 western events the FE averaged magnitude A0 was $4.5 \%$, maximum value of the equatorial component of anisotropy Axy $=3.5 \%$, solar wind velocity did not exceed $600 \mathrm{~km} / \mathrm{s}$. For 10 eastern events the mean value of $\mathrm{A} 0=4.2 \%$, maximum $\mathrm{Axy}=2.1 \%$.

In Fig. 4 vector diagrams of the anisotropy Axy are presented comparing the eastern and western events. We can note very specific behavior of the CR anisotropy before and during the FEs which is different for eastern and western events. By an order of magnitude the increase of Axy is essentially larger for western FEs, however for 'east' events we observe sharp changes of direction of Axy during the main phase of Forbusheffect. The properties of Axy behavior may help with the identification of a disturbance source causing the FE when there is not sufficient information to make it unambiguously (e.g. Fig. 5). In these plots one can see the characteristic behavior of Axy vector which evidences the FE is caused by a western source.

All the above considered FEs are associated with disturbances originating from sources far from Sun center, and every time the Earth was localized at the edge of the propagating 

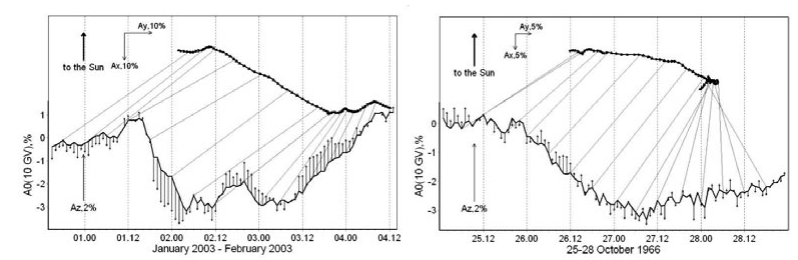

Figure 5. Two examples of events from non-identified sources (February 2003 and October 1966).

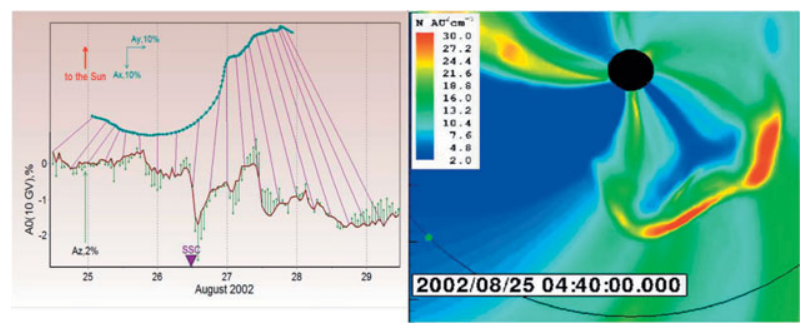

Figure 6. Vector diagram of CR anisotropy behavior and CME model calculations (Lugaz et al. 2008) during the August 2002 event.

disturbance, the near-Earth measurements did not show a high perturbation in the Earth's vicinity. The high magnitude of FEs from such remote sources on this quiet background implies great power of a disturbance and tells about modulating over an area wider than the size of the disturbance. How this may occur in the case of a western source may be, in particular, illustrated by Fig. 6 where the picture of propagating disturbance (adopted from Lugaz et al. 2008) and behavior of CR anisotropy are presented together.

The FE started on August 26, 2002 after weak shock arrival on the background of moderate interplanetary and geomagnetic activity $\left(\mathrm{B}_{m}=15 \mathrm{nT}, \mathrm{Kp}=5-\right.$, minimum $\mathrm{Dst}=-47$ $\mathrm{nT}$ ). The FE magnitude was not large (about $2 \%$ ), but the CR anisotropy was rather large for such small effect $-3.3 \%$. It was caused by a CME originating from the western limb (W81) and associated with X3.1/1F solar flare. Fig. 6 demonstrates that the western flank of the CME came to open magnetic field lines connected to the Earth. This effect is one of the reasons why this CME originating from the western limb caused a noticeable FE with large CR anisotropy on the unsettled background.

\section{Conclusions}

- In the majority of effective events the Earth enters only a periphery of the interplanetary disturbance, the main part of which misses Earth (to the East or to the West).

- Those events are preceded by powerful flares on the Sun, which are generally located far from the center of the solar disk. The CMEs and interplanetary disturbances, originating from the near limb longitudes, appear to be of larger size and more complicated structure than is visible near Earth.

- It was possible to separate small but very definite groups of large FEs which were not followed by strong interplanetary disturbances near Earth, nor by high geomagnetic activity but seemed to be associated with great solar wind disturbances that missed Earth. 
- The anomalous eastern FEs have a prolonged descent phase with a later minimum but larger magnitude in CR density than western events. They show a sharp change of the anisotropy direction in the minimum of FE.

- The anomalous western FEs strongly differ from typical FEs caused by western sources which are usually not large and very short. They also differ from the anomalous eastern FEs by a bigger size of CR anisotropy and less variability of its direction.

- These properties may be used for the inner heliosphere as diagnostics and Space Weather predictions.

- In the cases of far sources the CR observations give better information about the real power and size of a disturbance than near-Earth measurements of the solar wind. Moreover, it may be a useful tool in the cases of events where there is not sufficient data for their identification.

\section{Acknowledgement}

This work is supported by RFBR Grants 07-02-00915, 07-02-13525, by the Program of Presidium RAS "Neutrino Physics". Authors are thankful for the collections from all stations of world networks providing the monitoring of the CR neutron component http://cr0.izmiran.rssi.ru/ThankYou/main.htm.

\section{References}

Asipenka, A. S., Belov, A. V., Eroshenko, E. F., Klepach, E. G., \& Yanke, V. G. 2008, J. Adv. Space Res., doi:10.1016/j.asr.2008.09.022

Belov, A. V., Gushchina, R. T., Eroshenko, E. A., Yudakhin, K. F., Yanke, \& V. G. 2007, Geomagnetism and Aeronomy, 47, 251

Belov, A. V., Gushchina, R. T., Eroshenko, E. A., Ivanus, D., \& Yanke, V. G. 2005, Proc. 29th Internat. Cosmic Ray Conf., 2, 239

Belov, A. V., Eroshenko, E. A., Struminsky, A. B., \& Yanke, V. G. 2001, Adv. Sp. Res., 27, 625

Belov, A., Bieber, J., Eroshenko, E. A., Evenson, P., Pyle, R., \& Yanke, V. G. 2003, Adv. Sp. Res., 31, 919

Belov, A. V., Buetikofer, R., Eroshenko, E. A., Flueckiger, E. O., Oleneva, V. A., \& Yanke V. G. 2003, Proc. 28th Internat. Cosmic Ray Conf., 6, 3581

Lugaz, N., Roussev, I. I., Sokolov I. V., \& Jacobs C. 2008, Proc. IAU275 Symposium, in this volume

Papaioannou, A., Belov, A., Mavromichalaki, H., Eroshenko, E., Oleneva, V. J., \& Yanke, V. G. 2008, J. Adv. Space Res., doi:10.1016/j.asr.2008.09.003

\section{Discussion}

Gopalswamy: In the anomalous FE events, the correlation is different because the maximum magnetic field used is not the real field in the ICME. If you estimate the correct $B_{\max }$, the anomalous FEs will look like the normal FE events.

Eroshenko: The anomalous FEs look so because we do not have a correct $B_{\text {max }}$ (it might be obtained either from the full observations or from any good model). Since we have only near Earth observations, it doesn't reflect the situation near (or inside) the disturbance and we are talking about "anomalous" effect with respect to data in the Earth environment. 